\title{
RECURRENCE OF DEFORMITY IN IDIOPATHIC CTEV TREATED BY PONSETI
} TECHNIQUE

\section{Orthopaedics}

\author{
Dr. Tushar kanti \\ Ghorai*

\section{ABSTRACT}

Congenital talipes equinovarus (CTEV) or clubfoot, is one of the commonest congenital deformity . At present,Ponseti method is most commonly used successful reliable, essentially conservative technique for correction of CTEV deformity and successful in upto $98 \%$ of feets. However there is a chance of the relapse or recurrence.

A prospective and observational study after getting institutional ethical clearance was conducted in the department of Orthopedics, at COM and JNM Hospital, Kalyani, Nadai, WB, between August 2018 to July , 2020 including baby with club foot . Total study sample size was 20 according to inclusion and exclusion criteria.

The mean age of presentation was $4 \pm 2.51$ week. The male to female ratio was $1.86: 1$. Bilateral feet involvement was $50 \%$. In unilateral involvement, right side was affected $70 \%$, left side was affected in $30 \%$ cases. Family history of CTEV was seen in $20 \%$ cases. Mean body weight of babies was $2.49 \pm 0.17 \mathrm{~kg}$. At presentation, mean pirani score was $5 \pm 0.48$. Tenotomy was done $75 \%$ cases . Relapse rate was $15 \%$, mean age of relapse was $7 \pm 1.73205$ month, occurred in most common in left side $(66 \%)$. There was history of non-adherence of abduction brace for $>2$ month in all three patients adduction score was 0.5 in all child. Most common deformity relapse was adduction of forefoot.

Ponseti method is cost effective especially in developing country. Strict adherence to brace protocol and good communication between heath care persons and parents reduces incidence of relapse. Relapse is relatively common even in Ponseti method and most of cases, it manageable by conservative treatment like cast.

\section{KEYWORDS}

CTEV, clubfoot, Ponseti method, Relapse, Recurrence

\section{Introduction :}

Congenital talipes equinovarus (CTEV), also known as clubfoot, is common congenital ${ }^{1}$ foot deformity in children characterized by hindfoot equinus, hindfoot varus, midfoot cavus, and forefoot adduction ${ }^{2}$. Although a number of conservative and surgical methods have been proposed to correct the clubfoot deformity, the relapses of the clubfoot are not uncommon. At present, Ponseti method is most commonly used successful reliable, quick ${ }^{3,4}$ non-operative technique for correction of CTEV deformity and successful in upto $98 \%$ of feets ${ }^{3}$ However there is a necessity to investigate the relapse ${ }^{5}$ or recurrence ${ }_{24,6}$ pattern, to achieve successful outcome without or reduce relapse.

\section{Review of literature:}

Congenital talipes equinovarus (CTEV) or clubfoot is one of the most common pediatric foot deformity, with estimated birth prevalence of 1 in 1000 live births ${ }_{1,4,7,8}$ and upto $50 \%$ cases ,it is bilateral involvement ${ }^{8}$. Although, there are a number of conservative or operative treatments have been used to correct the clubfoot, it is still challenging to treat the most severe cases of clubfoot. For the last 150 years, the treatment methods used for clubfoot are still controversial Because, the extensive surgical procedures (repeated soft tissue releases) on the clubfoot lead to induce some complications such as stiffness of foot, arthritic problems ${ }^{9}$ and poor quality of life. After that, a number of non-operative ${ }^{10}$ methods are proposed to correct the clubfoot deformity with the following techniques such as different methods of manipulations, orthosis or splinting or bracing, casting, and strapping. Historically, conservative management was introduced by Hippocrates in around $400 \mathrm{BC}$. Later, Kiteintroduced his method, referred as Kite method ${ }^{4,10}$, which is including manipulation and casting technique, but the success rate of this method was poor Subsequently, Ponseti developed a conservative method, called as Ponseti method ${ }^{3,10}$, with manipulation, casting, Achilles tenotomy and bracing, and it takes about four to five weeks to achieve the full correction of all four components of the clubfoot deformity. In this method, Achilles tenotomy is used to release the equinus deformity and bracing for maintaining the corrected clubfoot, and it helps to obtain the supple ${ }^{6}$, plantigrade, functional, pain-free foot ${ }^{3,410}$. Initial treatment for clubfoot should be a conservative method to correct the clubfoot successfully, the relapses ${ }^{5}$ is still not avoidable. Despite several studies reported that Ponseti techniques provide more successful results, the clubfoot relapses or recurrences of the clubfoot can be still seen in the children with less than 2 years of age followed by the Ponseti method of treatment. Adherence of Ponseti protocol is necessary to achieve full successful clubfoot treatment without relapses of any deformities. Several literatures review articles reported the technical details and adherence of Ponseti protocol among walking age or older children. There is no definite relapse pattern of the clubfoot, causative factors for relapses. Objectives of this study is to assess recurrence of deformity in idiopathic CTEV treated by Ponseti technique in the term of type of deformity recurrence , severity of deformity, its timing of appearance

\section{Material and Methods :}

The study was a prospective and observational study conducted in the department of Orthopedics, at COM and JNM Hospital, Kalyani, Nadai, WB, between August 2018 to July , 2020 including baby with club foot. Approval by the ethics committee of COM and JNM Hospital, kalyani had taken( Ref.No. : F-79/Pr/COMJNMH/S.R.C.15 dated 17.07.2018) and written informed consent of patients of babies obtained to conduct the study. Total study sample size was 20 according to inclusion and exclusion criteria.

\section{Inclusion criteria:}

Age : $<3$ month infants

CTEV foot deformity

Not treated previously

\section{Exclusion criteria:}

CTEV with congenital anomaly or syndrome or neurological causes of club foot like arthrogryposis multiplex congenital, congenital myotonic dystrophy, myelomenigocele, amniotic band sequence ,spina bifida At visit at orthopaedics OPD of COM \& JNM Hospital , $<3$ month old infant with foot deformity was clinically assessed CTEV deformity.If present, child was assessed for any other syndrome like AMC , menigomyelocele, spina bifida, sign of spinal dysmorfism . If any syndrome/ congenital anomaly was not detected clinically, selected for study as idiopathic CTEV, after getting consent from patents of infant. Then this CTEV child was assessed clinically, Pirani scoring was done, patients demography recorded. Serial manipulation and casting $1 \mathrm{wk}$ interval done. After correction of cavus, adduction deformity was corrected. After correction of cavus and adduction deformity ,if dorsiflexion $15^{\circ}$ was not possible passively, percutaneous TA tenotomy for residual eqinus deformity correction was done and 3 week POP cast to be applied according to standard Ponseti technique.

After correction of deformities and after removal of last plaster, Abduction brace like Steinbeck shoe was applied for $23 \mathrm{hr} / 24 \mathrm{hr}$ for first 3 month , then 12-14 hr/day. Abduction brace size was changed according to size of foot up to 1 year. 
After initial full correction of deformity, each child was assessed serially at $3^{\text {rd }}, 6^{\text {th }}, 9^{\text {th }}$ month, $1 \mathrm{yr}$ for pirani score , relapse pattern, severity, time of relapse.

Non-compliance to abduction brace was defined as the inability to adhere to the above mentioned criteria and also delay in changing the splint and shoes as the foot size changed. The parents were instructed to perform range of motion exercises for the ankle and foot when it was out of the brace.

Statistical analysis : The collected data will be organized, tabulated and statistically analysis using IBM,SPSS Statistic for Window version 22. Continous variable will be expressed as mean \pm standard deviation (sd). For categorical data, frequency was calculated.

\section{RESULTS:}

A total 20 children ( 30 idiopathic club feet) was follow up from August 2018 to July 2020 . Each child has been follow up over 1 year from the initiation of treatment. There was no lost follow up. The mean age of presentation was $4 \pm 2.51$ week . The male to female ratio was 1.86:1. Bilateral feet involvement was $50 \%$. In unilateral involvement, right side was affected $70 \%$, left side was affected in $30 \%$ cases. Family history of CTEV was seen in $20 \%$ cases. Mean body weight of babies was $2.49 \pm 0.17 \mathrm{~kg}$. At presentation, mean pirani score was $5 \pm$ 0.48 . Tenotomy was done $75 \%$ cases and mean pirani score before tenotomy was $1.67 \pm 0.24$. Mean pirani score after 3 month of treatment was $0.2 \pm 0.25$. Mean plaster required was $5 \pm 0.732$.

Pearson correlation efficient (r) between pirani score at presentation and $3^{\text {rd }}$ month of treatment was 0.325 i.e positive correlation . Mean pirani score of tenotomy patients ( 15 children) was $5 \pm 0.46$. Mean pirani score of non-tenotomy children ( 5 children ) was $4.5 \pm 0.38$. Pearson coefficient correlation between body weight and pirani score was $r=0.2001$ i.e. positive correlation but it is weak. Compare to preand post treatment pirani score using paired t- test significant improvement ( The t-value is 38.38222. The $\mathrm{p}$-value is $<.00001$ ).

Initial correction after 1year of follow up of each child, success rate of Ponseti method in our study is $85 \%$.

Relapse rate was $15 \%$, mean age of relapse was $7 \pm 1.73205$ month, occurred in most common in left side (66\%). There was history of nonadherence of abduction brace for $>2$ month in all three patients adduction score was 0.5 in all child. Most common deformity relapse was adduction of forefoot. Additional 2 cast was applied for 2 patients, after 2 plaster, one patient was operated for adduction and vaus after 1 yr of age.

\section{DISCUSSION :}

Ponseti method is essentially a conservative method , it gives $>90 \%$ successful long term result in idiopathic clubfoot ${ }^{9,11}$. Club foot has inherent tendency to relapse irrespective of treatment protocol. Rate of relapse varies upto $56 \%$ by various authors ${ }^{12}$. Cause of relapse is noncompliance to abduction brace $e^{1,4,11,12,13,14,15}$, severity of initial deformity ${ }^{6}$, calf muscle atrophy, waek muscle around ankle ${ }^{16}$, parental education ${ }^{15,17}$, female gender ${ }^{17}$, higher pre-treatment score ${ }^{17}$, . But there is no universal definition of non-compliance of abduction brace. In this study, mean age of presentation was 4 week, , male child mostly affected ,bilateral involvement was in $50 \%$ cases. Changulani ${ }^{2}$ et al showed that bilateral involvement in $52 \%$. Ponseti IV, Smoley $\mathrm{EN}^{6}$ showed in their study bilateral involvement in $40 \%$ cases. Chand $\mathrm{S}^{11}$ et al showed that in unilateral case, right side affected more than left side of foot. In our study, family history of CTEV was in $20 \%$ case . Rasit A et al ${ }^{8}$ showed that family history was seen in $24.4 \%$ cases.

In our study, mean body weight of babies was $2.49 \mathrm{~kg}$ and mean pirani score was 5 . Changulani ${ }^{2}$ et al showed that pirani score at presentation was 5 .

Tenotomy was done $75 \%$ cases. Changulani ${ }^{2}$ et al showed that tenotomy was done $85 \%$ cases. Dyer PJ, Davis $\mathrm{N}^{3}$ showed that tenotomy was done $60 \%$ cases, Sharma $\mathrm{A}^{18}$ showed that tenotomy was done in $76.3 \%$.

Mean plaster was required 5 in our study. Boehm $\mathrm{S}^{1}$ in their study showed that cast in idiopathic club foot group was applied $4.5 \pm 1.2$. score was positive. Dyer PJ, Davis $\mathrm{N}^{3}$ showed that initial pirani score and number of cast required was positive correlation using chi-square test.

In our study, success rate for correction of deformity in Ponseti method is $85 \%$. Porecha MM et al ${ }^{10}$ showed in their study success rate $90 \%$ and Zhao $\mathrm{D}^{12}$ stated that initial correction rate was $89.2 \%$.

Relapse rate was $15 \%$ at the mean age of 7 month and adduction of fore foot was common deformity that relapse in our study. Relapse was adduction ( pirani score 0.5 in 3 child) and vaus was 0.5 (one child). Boehm S showed that in idiopathic club foot group relapse was in 6 feet out of 22 feet. Changulani ${ }^{2}$ et al showed that relapse was $32 \%$ due to lack of compliance to splint. Chand $\mathrm{S}^{11}$ showed that most common relapse was equinus. Ganesan $\mathrm{B}^{14}$ showed that most common relapse was adduction. Relapse occurs singularly or more commonly in various combination

Abduction brace is mandatory to prevent relapse ${ }^{15}$.Still relapse has proven to be less rigid and easier to treat ${ }^{19}$.

Limitation of study: In bilateral clubfeet, only higher pirani score of foot was used as pirani score of child . In our study sample size was small , short follow up period too short.

In conclusion, Ponseti method is cost effective especially in developing country. Strict adherence to brace protocol and good communication between heath care persons and parents reduces incidence of relapse. Relapse is relatively common even in Ponseti method and most of cases, it manageable by conservative treatment like cast.

\section{REFERENCES}

1. Boehm S, Limpaphayom N, Alaee F, Sinclair MF, Dobbs MB. Early results of the Ponseti method for the treatment of clubfoot in distal arthrogryposis. J Bone Joint Surg Am. 2008;90:1501-1507.

2. 2. Changulani M, Garg NK, Rajagopal TS, Bass A, Nayagam SN, Sampath J, Bruce CE. Treatment of idiopathic club foot using the Ponseti method: initial experience. J Bone Joint Surg Br. 2006;88:1385-1387

3. Dyer PJ, Davis N. The role of the Pirani scoring system in the management of club foot by the Ponseti method. J Bone Joint Surg Br. 2006;88:1082-1084

4. Jowett CR, Morcuende JA, Ramachandran M. Management of congenital talipes equinovarus using the Ponseti method. a systematic review. J Bone Joint Surg Br 2011;93:1160-1164

5. Ponseti IV Relapsing clubfoot: causes, prevention, and treatment. Iowa Orthop J. 2002;22:55-56.

6. Ponseti IV, Smoley EN. The classic: congenital club foot: the results of treatment. 1963 Clin Orthop Relat Res. 2009;467: 1133-1145.

7. Charak SS, Muzafar K, Singh O. Management of idiopathic clubfoot with Ponset technique. Int J Res Med Sci 2017;5:3355-8.

8. Rasit A, Rasit A, Azani H, Zabidah P, Merikan A, Nur Alyana B. Clubfoot: the treatmen outcome using quantitative assessment of deformity. Malays Orthop J. 2012:6(SupplA):2-5. doi:10.5704/MOJ.1211.002

9. Bor N, Coplan JA, Herzenberg JE. Ponseti treatment for idiopathic clubfoot: minimum 5-year followup. Clin Orthop Relat Res. 2009:467(5):1263-1270 doi:10.1007/s11999008-0683-8

10. Porecha MM, Parmar DS, Chavda HR. Mid-term results of Ponseti method for the treatment of congenital idiopathic clubfoot: a study of 67 clubfeet with mean five yea follow-up. J Orthop Surg Res. 2011;6:3

11. Chand S, Mehtani A, Sud A, Prakash J, Sinha A, Agnihotri A. Relapse following use of Ponseti method in idiopathic clubfoot. J Child Orthop. 2018;12(6):566-574 doi:10.1302/1863-2548.12.180117

12. Zhao D, Li H, Zhao L, Liu J, Wu Z, Jin F. Results of clubfoot management using the Ponseti method: do the details matter? A systematic review. Clin Orthop Relat Res. 2014;472(4): 1329-1336 doi:10.1007/s11999-014-3463-7

13. Harnett P, Freeman R, Harrison WJ, Brown LC, Beckles V. An accelerated Ponseti versus the standard Ponseti method: a prospective randomised controlled trial. J Bone Joint Surg Br. 2011;93(3):404-408. doi:10.1302/0301-620X.93B3.24450

14. Ganesan B, Luximon A, Al-Jumaily A, Balasankar SK, Naik GR. Ponseti method in the management of clubfoot under 2 years of age: A systematic review. PLoS One. 2017;12(6):e0178299. Published 2017 Jun 20. doi:10.1371/journal.pone.0178299

15. Siapkara A, Duncan R. Congenital talipes equinovarus: a review of curren management. J Bone Joint Surg Br. 2007;89(8):995-1000. doi:10.1302/0301620X.89B8.19008

16. Dunkley M, Gelfer Y, Jackson D, et al. Mid-term results of a physiotherapist-led Ponseti service for the management of non-idiopathic and idiopathic clubfoot. J Child Orthop. 2015;9(3):183-189. doi:10.1007/s11832-015-0658-8

17. Mahan ST, Spencer SA, May CJ, Prete VI, Kasser JR. Clubfoot relapse: doe presentation differ based on age at initial relapse? J Child Orthop. 2017;11(5):367-372 doi:10.1302/1863-2548.11.170016

18. Sharma A, Shukla S, Kiran B, Michail S, Agashe M. Can the Pirani Score Predict the Number of Casts and the Need for Tenotomy in the Management of Clubfoot by the Ponseti Method? Malays Orthop J. 2018;12(1):26-30. doi:10.5704/MOJ.1803.005

19. Sætersdal C, Fevang JM, Fosse L, Engesæter LB. Good results with the Ponseti method: a multicenter study of 162 clubfeet followed for $2-5$ years. Acta Orthop. 2012;83:288-293. 